\title{
A 7-year Prospective Epidemiological Study of Acute Elbow Admissions to a Trauma Unit
}

\author{
Alex Bolt ${ }^{1,2}$, Siddharth Govilkar ${ }^{1,2}$, Caroline Dover ${ }^{1,2, ~ *, ~ J o h n ~ B l a c k w e l l ~}{ }^{1,2}$, Stuart Hay ${ }^{1,2}$ \\ ${ }^{1}$ Trauma and Orthopaedics, The Robert Jones and Agnes Hunt Hospital, Gobowen, Oswestry, UK \\ ${ }^{2}$ Trauma and Orthopaedics, Shrewsbury and Telford Hospitals NHS Trust, Shrewsbury, Shropshire, UK
}

Email address:

bolta1@doctors.org.uk (A. Bolt), siddoc1984@yahoo.co.uk (S. Govilkar), c.dover@doctors.org.uk (C. Dover), jrblackwell@doctors.org.uk (J. Blackwell), stuarthay@doctors.org.uk (S. Hay)

${ }^{*}$ Corresponding author

\section{To cite this article:}

Alex Bolt, Siddharth Govilkar, Caroline Dover, John Blackwell, Stuart Hay. A 7-year Prospective Epidemiological Study of Acute Elbow Admissions to a Trauma Unit. Journal of Surgery. Vol. 8, No. 6, 2020, pp. 190-196. doi: 10.11648/j.js.20200806.14

Received: September 28, 2020; Accepted: October 16, 2020; Published: November 11, 2020

\begin{abstract}
Introduction: This study represents an analysis of data collected prospectively over a seven-year period to our trauma unit, to identify the epidemiological background behind the admissions of patients with acute elbow problems and to highlight the impact of these admissions on our trauma service. Method: Nearly 16,000 patients were included in our database. The admissions were initially categorized into A) fractures/dislocations or B) soft tissue trauma. The fractures/dislocations were then further classified by radiographic anatomical location, with data also collected on length of stay and operative management. In total 509 admissions were included in this study; 193 were paediatric cases and 316 were adults. This included 428 admissions with fractures/dislocations and 81 cases of soft tissue trauma. Conclusion: In conclusion, this data allows us to identify relative frequency of acute elbow conditions, including the incidence of common fracture patterns. These figures help to facilitate resource planning, such as expertise amongst personnel or the acquisition of equipment, including radial head replacement, elbow arthroplasty, and periarticular locking plates.
\end{abstract}

Keywords: Elbow Fracture, Epidemiology, Supracondylar Fracture, Paediatric Elbow, Dislocation, Elbow Dislocation, Elbow Trauma

\section{Introduction}

The primary activity of the elbow, in conjunction with the shoulder, is to position the hand in space so that it can, in turn, deliver prehensile function. However, the elbow is often quite sensitive and unforgiving following physical insult, which can result in stiffness, pain, instability and locking, which will inevitably impact upon function.

The range of motion required to perform most activities of daily living (ADLs) is through a functional flexion/extension arc of $100^{\circ}$, often quoted as $30^{\circ}-130{ }^{\circ}$ with $50^{\circ}$ of pronation \& supination [1].

The acute elbow is a frequent presentation to the emergency department, with a UK district general hospital expecting to see over 400 acute elbows in a 9-12 month period [2]. A proportion of these patients require inpatient care and it is important to understand both the mechanism of injury and the implications for service provision which accompany this.

It is well documented amongst the paediatric population that supracondylar fractures of the humerus are the most common injury and may account for two-thirds of all hospital admissions for paediatric elbow injuries [3-5]. However, amongst adults the reasons for admission are more variable, including a large variety of acute fractures and soft tissue trauma that requires fixation or repair.

It is important that those hospitals treating the acute elbow have the appropriate equipment and expertise at their disposal, particularly given the potential complexity of injury and the residual morbidity, which may include stiffness and ectopic ossification. We have identified both the pathology and the management associated with the acute elbow admissions over a 7-year period. The numbers are large and therefore provide a genuine reflection of these admissions, serving a local population of 300,000 . Besides being of general interest to the upper limb surgeon, it potentially 
provides information for the planning of an elbow service within the acute setting.

\section{Materials \& Methods}

An admissions database of almost 16,000 patients was collected prospectively over a 7-year period at a district general hospital, which provides a regional trauma unit. This database was reviewed by two independent orthopaedic personnel in conjunction with radiographs and their reports, in confirmation of acute elbow pathology.

All acute elbows were identified and categorized into "Fractures/Dislocations" or "Soft Tissue Trauma". Using the available radiographs, the fractures/dislocations were then sub-classified by bone type. All Monteggia, radio-capitellar and ulna-trochlea dislocations were included. Our definition of "elbow fracture" identified the supracondylar fracture as the proximal limit. Fractures both including, and distal to, the supracondylar territory were therefore included within this study. Fractures distal to the coronoid of the ulna or radial neck were excluded, unless part of a Monteggia fracture dislocation or a Galeazzi fracture. Therefore, all fractures between these proximal and distal limits qualified for inclusion. Data was also collected on 1) length of stay, 2) whether the patient required operative management, and 3) the type of surgery performed.

All statistical analysis and graphical representation of data was performed in "Microsoft Excel" 2008 version 12.3.6. Chi square test was used to compare categorical variables and a $\mathrm{p}$-value of $<0.05$ was considered significant.

\section{Results}

In total 509 admissions were identified in this study, which included 428 admissions with fractures/dislocations and 81 cases of soft tissue trauma. There were 316 adult admissions and 193 paediatric admissions, with a median age of 58 years amongst the adults (range $17-99$ ), and mean age of 8 years within the paediatric cohort (range $1-16$ ). Admissions varied by season as shown in Figure 1 .

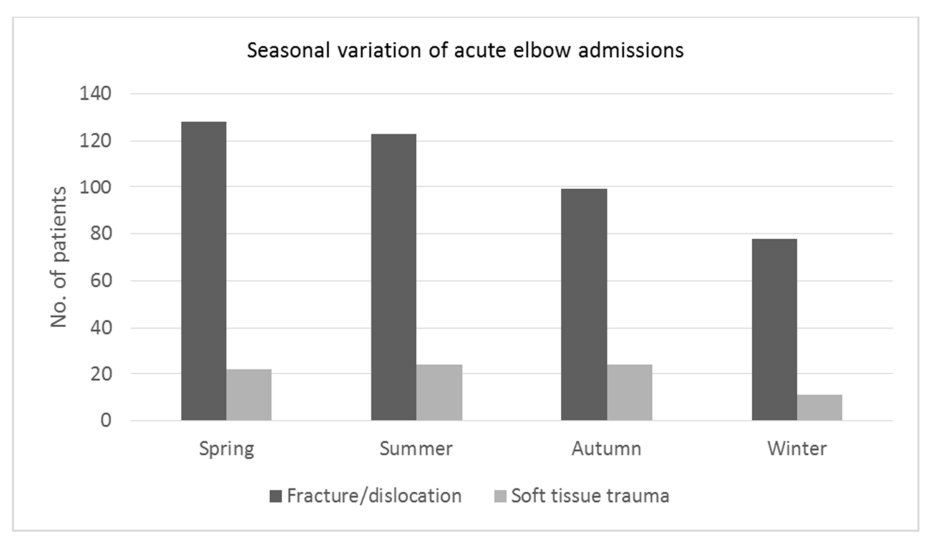

Figure 1. Seasonal variation of acute elbow admissions.

\subsection{Fractures}

\subsubsection{Paediatric Fracture Group}

An isolated supracondylar fracture was the most common reason for admission within the paediatric group, accounting for 100 of the 193 admissions. 85 of these required surgical intervention, of which 76 had K-wire fixation and 8 had closed manipulations. One of the 85 patients had an open reduction and internal fixation using a locking plate. The majority of these fractures occurred in spring, with less than half as many in winter. Their annual distribution is shown in Figure 2.

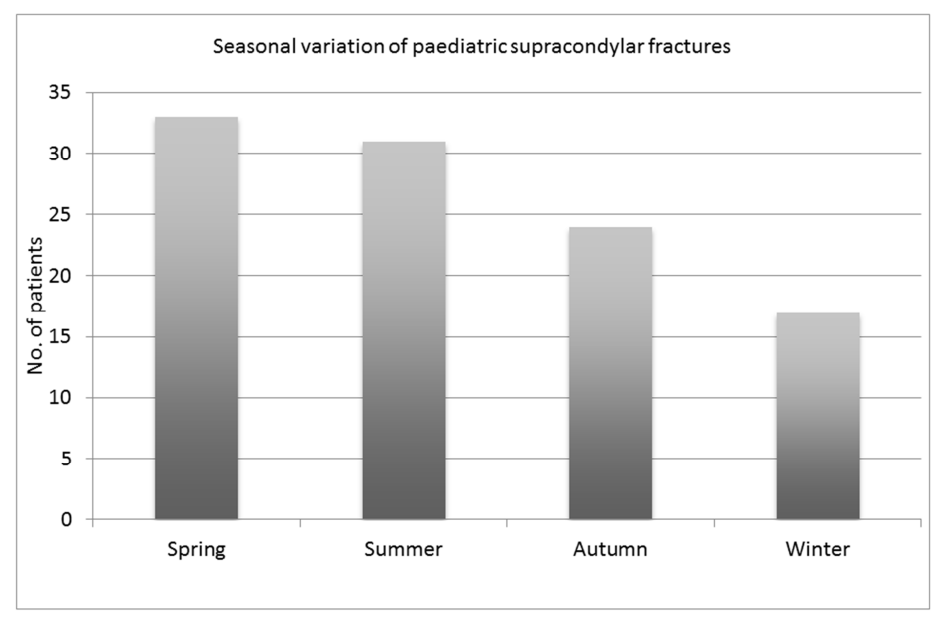

Figure 2. Seasonal variation in supracondylar fractures. 
A chi square test demonstrated no statistically significant difference in supracondylar fracture admissions between the seasons, including when grouped spring/summer and autumn/winter ( $p$ value $=0.49$ ) or when individual seasons were directly compared ( $\mathrm{p}$ value $=0.63$ ).

The distribution of isolated fracture types within our paediatric series is shown in Table 1.

Amongst the paediatric cohort there were only 2 capitellar and 4 trochlea fractures, and these all occurred in association with other fractures. In total there were 9 cases of multiple fractures around the elbow within our paediatric group. The cases of multiple fracture, in the absence of dislocation, and their subsequent management are presented in Table 2.
Table 1. Number of paediatric patients with isolated fractures in our series and their respective fractures.

\begin{tabular}{ll}
\hline Fracture & Number of patients \\
\hline Supracondylar & 100 \\
Lateral epicondyle & 12 \\
Monteggia & 11 \\
Olecranon & 8 \\
Radial head & 6 \\
Medial epicondyle & 3 \\
Radial neck & 3 \\
Coronoid & 1 \\
Total & 144 \\
\hline
\end{tabular}

Table 2. Cases of multiple elbow fractures in paediatric patients, in the absence of dislocation.

\begin{tabular}{|c|c|c|c|c|c|c|}
\hline Case number & Age (years) & Fractures & & & & Management \\
\hline 1 & 5 & Capitellum & Lateral epicondyle & & & K-wire fixation \\
\hline 2 & 11 & Capitellum & Trochlea & & & Headless compression screws \& locking plate \\
\hline 3 & 8 & Lateral epicondyle & Capitellum & & & K-wire fixation \\
\hline 4 & 12 & Medial epicondyle & Radial head & Radial neck & & K-wire fixation \\
\hline 5 & 6 & Monteggia & Radial neck & & & Manipulation under anaesthesia \\
\hline 6 & 11 & Supracondylar & Medial epicondyle & Lateral epicondyle & Trochlea & K-wire fixation \\
\hline 7 & 10 & Supracondylar & Lateral epicondyle & Trochlea & & Screw fixation \\
\hline 9 & 15 & Supracondylar & Medial epicondyle & Lateral epicondyle & Trochlea & Screw fixation \\
\hline
\end{tabular}

\subsubsection{Adult Fracture Group}

Amongst the adult cohort, the most common isolated fracture was that of the olecranon, accounting for 89 admissions. Twenty-nine of these patients (33\%) were managed with a locking plate and two with a standard plate. Twenty-eight patients received tension band wiring (31\%). Twenty-seven were managed conservatively and two of these patients had a manipulation under anaesthesia prior to this decision. The distribution of isolated fractures within our adult series is shown in Table 3.

Table 3. Number of adult patients with isolated fractures in our series and their respective fractures.

\begin{tabular}{ll}
\hline Fracture & Number of patients \\
\hline Olecranon & 89 \\
Radial head & 22 \\
Supracondylar & 14 \\
Capitellum & 7 \\
Monteggia & 4 \\
Medial epicondyle & 3 \\
Coronoid & 2 \\
Radial neck & 1 \\
Lateral epicondyle & 1 \\
Total & 143 \\
\hline
\end{tabular}

The cases of "multiple fractures" occurring in the absence of dislocation were more numerous amongst the adult population than within the paediatric group. We were unable to identify any common associations between fractures in this group.

\subsection{Elbow Dislocations}

There were 49 simple elbow dislocations, of which 16 occurred in the paediatric age group and 33 amongst the adults. All simple elbow dislocations were reduced closed and did not require further acute stabilisation. There was a total of 40 cases of complex elbow fracture-dislocations, which included 15 paediatric cases and 35 adults. The most common associated fracture within the paediatric group was that of the medial epicondyle, of which there were 14 . The single remaining complex paediatric fracture-dislocation involved fracture of the radial head. Interestingly, radial head fracture was the most commonly associated fracture amongst the adult group, of which there were 15 .

The one paediatric radial head fracture associated with elbow dislocation was stabilised with $\mathrm{k}$-wire fixation perpendicular to the fracture. Amongst the adults, 4 of the 15 radial head fractures required radial head replacement, one of which was supplemented with a bridging external fixator due to persistent elbow instability. Four of the other adult radial head fractures required acute fixation to provide elbow stability; three with headless compression screws and one with small fragment screws. The remaining dislocations with associated radial head fractures had manipulation under anaesthesia and casting.

The distribution of fracture-types associated with elbow dislocation amongst the adults are listed below in Table 4.

Table 4. Number of fractures that occurred in adults as part of a complex elbow fracture-dislocation.

\begin{tabular}{ll}
\hline Fracture & Number of patients \\
\hline Radial head & 15 \\
Coronoid & 8 \\
Lateral epicondyle & 4 \\
Capitellum & 4 \\
\hline
\end{tabular}




\begin{tabular}{ll}
\hline Fracture & Number of patients \\
\hline Olecranon & 2 \\
Medial epicondyle & 2 \\
Radial neck & 2 \\
Trochlea & 1 \\
\hline
\end{tabular}

Within the adult complex fracture-dislocation group multiple fractures were common, which is in contrast to the paediatric patients, where the associated fractures were isolated in each case. An example of a complex fracturedislocation from our series, managed conservatively, is shown in Figure 3 and Figure 4.

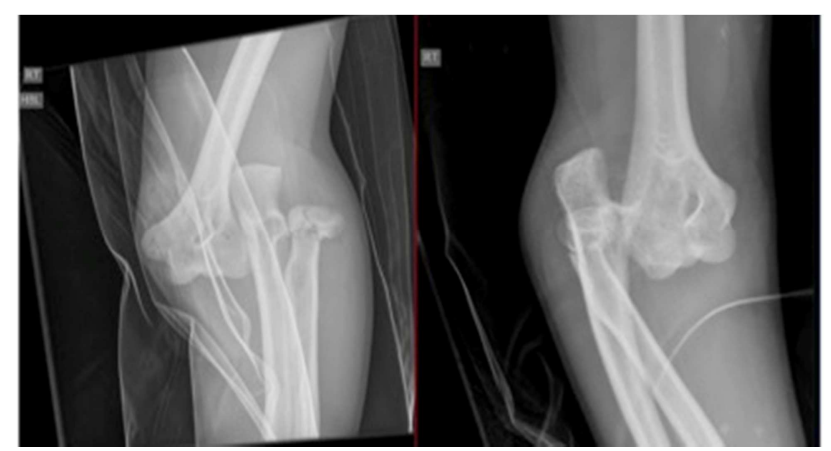

Figure 3. Complex fracture-dislocation in an adult patient with radial head and neck fractures.

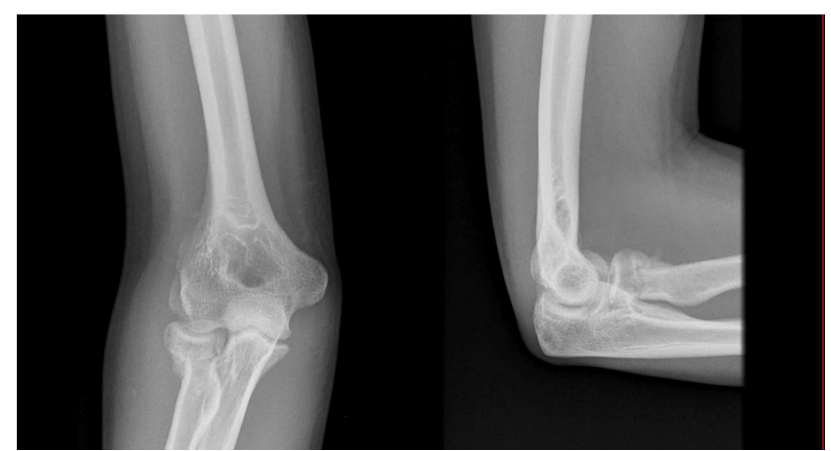

Figure 4. Follow up at 6 months of fracture-dislocation shown in figure 3.

Twelve of the 15 paediatric patients required acute fixation of their fractures to secure elbow stability. This was achieved by the use of simple K-wires and screws. An example of a complex paediatric fracture-dislocation is demonstrated in Figure 5 and Figure 6.

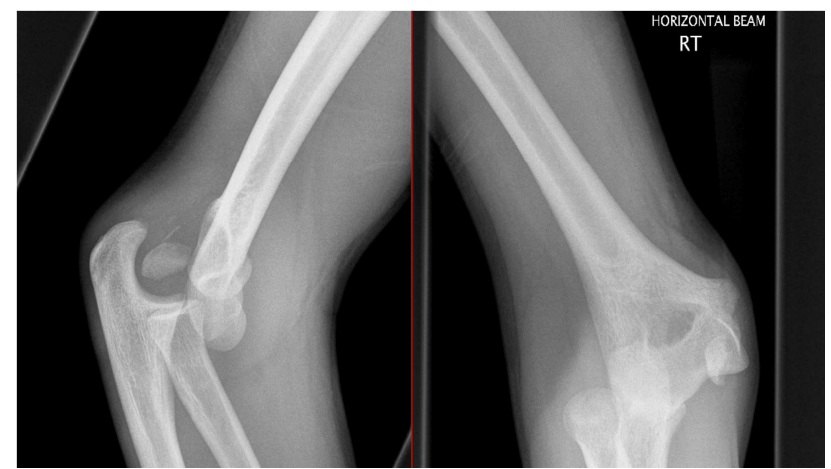

Figure 5. Paediatric complex fracture dislocation with medial epicondyle fracture.

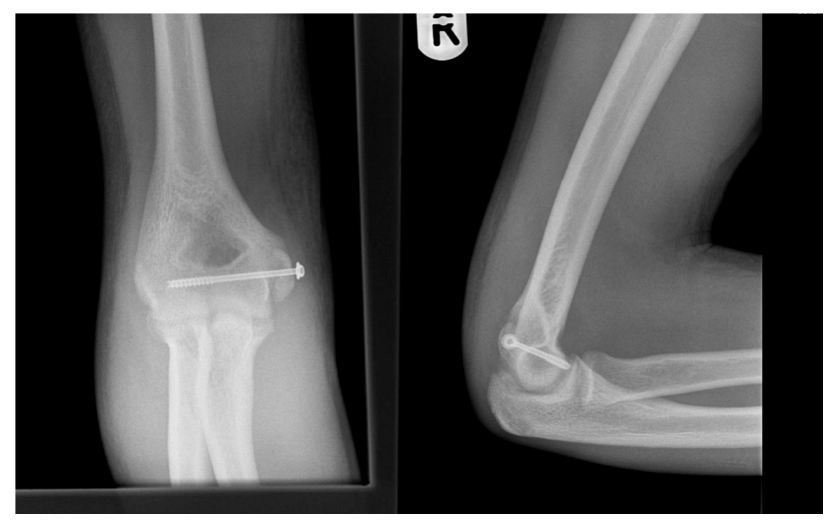

Figure 6. Follow up at 2 weeks with fixation in situ.

Only 8 of the 25 adult cases were managed by acute stabilization, but this required a larger range of equipment due to fracture complexity. It included radial head replacements, headless compression screws and bridging external fixation, in addition to standard screws and locking plates. One of the adult dislocations, initially managed conservatively, subsequently underwent total elbow arthroplasty 5 months following their injury, for established fracture non-union of the humerus. This was a supracondylar fracture pattern, which is notoriously associated with nonunion.

A variety of equipment and implants were used to manage the 428 patients (adult and paediatric) who had a combination of fractures, dislocations or both. The equipment is documented in table 5.

Table 5. Number and type of implants required to manage acute elbow injuries in our series.

\begin{tabular}{ll}
\hline Equipment & Number of patients \\
\hline K-wires & 101 \\
Locking plate & 90 \\
Tension band wire & 36 \\
Headless compression screws & 23 \\
Standard Screw (s) & 11 \\
Radial head replacement & 11 \\
Standard plate & 5 \\
Total elbow arthroplasty & 3 \\
External fixation & 1 \\
Flexible IM nails & 1 \\
\hline
\end{tabular}

The number of cases that required equipment/implants does not directly equate to the number of admissions for fractures and dislocations, due to some admissions being managed conservatively or by closed manipulation.

\subsection{Soft Tissue Trauma}

Eighty-one patients were admitted following soft tissue trauma to the elbow, with seventy-seven requiring surgical wound management. There were no neurovascular injuries. However, some penetrated into the joint and the majority involved the olecranon bursa. Soft tissue trauma admissions occurred much less frequently in winter months, with half as many admissions compared to spring. A chi-square test demonstrated that there was no statistically significant 
difference between the seasons with a $\mathrm{p}$ value of 0.59 .

\section{Discussion}

\subsection{Fractures}

The distribution of paediatric fractures within our series is consistent with current literature, with supracondylar fracture being the most common reason for admission [3, 4]. Our work demonstrates that elbow fractures amongst paediatric and adult populations occurred more commonly during the spring and summer, although this was not statistically significant. This is consistent with other published literature showing that paediatric supracondylar fractures are more common during the school holidays [6]. In keeping with our data, paediatric supracondylar fractures are acknowledged to be more common in the spring than in the summer [6].

Interestingly geographical location is recognised to affect the risk of paediatric fracture. Patients in Wales have a significantly greater incidence of fracture than those in Greater London [7]. Boys have greater incidence of fracture than girls, and this is generally attributed to greater risktaking behaviour amongst boys [7].

We did not collect data on the specific mechanism of injury in our series, but the majority of supracondylar fractures are due to falls from play equipment [6]. Trampolines are accountable for almost half, with monkey bars and climbing frames making up the majority of the remainder [6]. A study looking at trampoline injuries showed that $74 \%$ of injuries occurred when 2 or more people were present on the trampoline, and the majority of patients (77\%) did not fall from the trampoline when sustaining their injury [8].

Those supracondylar fractures which did not involve play equipment were usually secondary to simple falls, but just less than $10 \%$ have been shown to be due to a fall from a bike or horse [6]. Weather conditions have been shown to influence admissions secondary to fracture. Sunshine correlates with more fracture admissions and rainfall with fewer [9]. This is logical given that the mechanism of injury most commonly involves play equipment or outdoor activities $[6,8]$. The reason fractures are more common in spring than summer is unclear, but we postulate it may be linked to the slightly lower temperature and presence of ice or dew on the ground, as well as on the play equipment.

Amongst the adult population our most common isolated fracture requiring admission was that of the olecranon. Although radial head fractures are more common than those of the olecranon, they rarely require admission [10]. Amongst the olecranon fractures in our series an equivalent number were managed with tension band wiring (TBW), plate fixation or conservatively. Frederic Pauwels first described the principle behind TBW in 1980 and it is commonly quoted as a cost efficient and simple procedure [11, 12]. However, this is contrary to current literature, with poor technique being cited as common, with complication rates documented as high $[12,13]$. We would have expected a trend towards plating rather than TBW as our series, although this was not the case. Throughout the series the distribution of fixation method for olecranon fractures was similar. Conventional plating in the olecranon has significant pitfalls due to the cancellous nature of the bone limiting sufficient screw torque, which is necessary for stability [14]. Locking plates rely on the plate screw interface to generate sufficient torque, thus making the type and quality of the bone less relevant in achieving stability [14]. Their popularity in fracture fixation is well recognised and so it is understandable that their use has become standard practice [14].

\subsection{Elbow Dislocations}

The elbow joint is the most commonly dislocated joint amongst paediatric patients and is the second most commonly dislocated joint in adults, after the shoulder [15]. The annual incidence of elbow dislocations is 6.1 per 100,000 in the literature, with complex fracture dislocations accounting for $26 \%$ of cases [16]. However, in our series $45 \%$ of the dislocations were complex which likely reflects a greater force at the time of injury.

It is well documented that simple dislocations are better managed by functional rehabilitation rather than prolonged immobilisation or surgery, and this is common practice in our unit $[16,17]$. Paediatric patients should be warned about the potential development of late cubitus valgus although functionally this does not appear to have a significant effect [18].

The management of acute complex elbow fracture dislocations is based on the principles of elbow stability. Primarily the joint surfaces are reconstructed then the soft tissue component is assessed and dealt with appropriately in accordance with the "Morrey" hierarchy of primary and secondary elbow stabilisers. Complex fracture dislocations, once reduced, do not necessarily impart "complex elbow instability", but it is paramount to assess stability in the management of these injuries [19]. In our series, where features of instability were present a fixation was performed. This was necessary in 12 paediatric fracture dislocations (11 medial epicondyle and 1 radial head). The medial epicondyle is recognized to be the most commonly associated fractures in paediatric elbow dislocations [20]. Our series demonstrates how the adult and paediatric patients differ in their fracture patterns when associated with elbow dislocation. Multiple fractures were common in adults whilst only isolated fractures were present in paediatric patients. The most common associated fractures amongst the adult dislocations, were of the radial head and the coronoid, which is consistent with other published literature [21].

\subsection{Theatre Equipment \& Data Quality}

An understanding of the epidemiology of acute elbow fractures and dislocations allows planning of theatre resources. Kirschner wires, plates and screws are standard sets, but more specialist equipment can be rationalised. In our series 3 headless compression screw sets were used per year 
with 1-2 radial head replacements being performed annually. The trust could consider if it is worth the expense to keep a radial head replacement on site or whether it should be borrowed when required.

There was on average less than 1 case of elbow arthroplasty for trauma per year in our series and unless elbow replacement is part of a normal, regular elective practice it is not necessary to have an arthroplasty set on site. Under these circumstances it can be ordered for a planned semi-elective case. However, the delivery of elbow replacement is currently in flux and it is likely that all elbow replacements, whether elective or traumatic, will be undertaken in specialist centres in the future [22].

The data for this study was acquired through radiograph and CT interpretation by two orthopaedic personnel, in conjunction with the radiologist's report. The fractures and their associated dislocations in this series all required admission and so were not subtle in nature. There is good evidence that fractures suggestive of elbow instability are identifiable on radiographs with moderate interobserver reliability and reasonable accuracy [23]. It is therefore unlikely that a significant fracture or dislocation was missed in this series.

\subsection{Soft Tissue Trauma}

The elbow region is vulnerable to soft tissue injury during falls, which can require formal wound washout and closure in theatre. 77 patients were admitted for wound management of the elbow area following trauma. This is a significant number, which should be accounted for in admission and theatre planning. The presentation was more common in warmer seasons with very few in winter, likely due to patients wearing thicker clothing that covered the elbows during winter months.

\section{Conclusion}

We have prospectively documented, and retrospectively analyzed, admissions of acute elbow injury to our unit. In conclusion, this data allows us to identify the range of fracture and dislocation patterns, in addition to the distribution of pathology associated with the acute elbow. It has also provided us with an important understanding of the breadth of elbow pathology requiring admission, and it is likely that this pattern is similar around the United Kingdom.

The methods of fixation used allow us to make recommendations regarding the provision of theatre equipment, with this data also providing us with insight into the significant impact of soft tissue trauma on our service.

\section{References}

[1] Morrey BF, Askew LJ, Chao EY. A biomechanical study of normal functional elbow motion. J Bone Jt Surg Am. 1981; 63 (6): 872-7.

[2] Lennon RI, Riyat MS, Hilliam R, Anathkrishnan G, Alderson
G. Can a normal range of elbow movement predict a normal elbow x ray? Emerg Med J. 2007 Feb; 24 (2): 86-88.

[3] Houshian S, Mehdi B, Larsen MS. The epidemiology of elbow fracture in children: analysis of 355 fractures, with special reference to supracondylar humerus fractures. J Orthop Sci. 2001; 6 (4): 312-5.

[4] Landin LA, Danielsson LG. Elbow fractures in children. An epidemiological analysis of 589 cases. Acta Orthop Scand. 1986 Aug; 57 (4): 309-12.

[5] Wilkins KE. Fractures and dislocations of the elbow region. In: Rockwood CA Jr, Wilkins KE, King RE, editors. Fractures in children. 3rd ed. Philadelphia: JB Lippincott; 1991, p. 526617.

[6] Barr LV. Paediatric supracondylar humeral fractures: epidemiology, mechanisms and incidence during school holidays. J Child Orthop. 2014 Mar; 8 (2): 167-170.

[7] Moon RJ, Harvey NC, Curtis EM, de Vries F, van Staa T, Cooper C. Ethnic and geographic variations in the epidemiology of childhood fractures in the United Kingdom. Bone. 2016 Apr; 85: 9-14.

[8] Nyted M, Drogset JO. Trampoline injuries. Br J Sports Med. 2006 Dec; 40 (12): 984-987.

[9] Ahmad M, Khatri M, Hildreth T, Roysam GS, Nanu AM. Influence of weather conditions in the incidence of admissions in an acute orthopaedic ward in a district general hospital. Orthopaedic Proceedings Mar 2006, 88-B (SUPP I) 171.

[10] Kodde IF, Kaas L, Flipsen M, van den Bekerom MPJ, Eygendaal D. Current concepts in the management of radial head fractures World J Orthop. 2015 Dec 18; 6 (11); 954-960.

[11] Pauwels F. Biomechanics of the locomotor apparatus: contributions on the functional anatomy of the locomotor apparatus. Berlin: Springer-Verlag; 1980.

[12] Schneider MM, Nowak TE, Bastian L, Katthagen JC, Isenberg J, Romens PM et al. Tension band wiring in olecranon fractures: the myth of technical simplicity and osteosynthetical perfection. Int Orthop. 2014 Apr; 38 (4): 847-855.

[13] John J, Miller D, Ford DJ, Hay SM, Cool P. Olecranon fractures: Tension band wire or plate fixation? Orthopaedic Proceedings. April 2009. vol. 91-B no. SUPP II 257.

[14] Kubiak EN, Fulkerson E, Strauss E, Egol K. The Evolution of Locked Plates. J. Bone Jt Surg. Am. 2006; 88: 189-200.

[15] Kuhn MA, Ross G. Acute elbow dislocations. Orthop Clin North Am. 2008; 39: 155-161.

[16] de Haan J, Schep NWL, Tuinebreijer WE, Patka P, den Hartog D. Simple elbow dislocations: a systematic review of the literature. Arch Orthop Trauma Surg. 2010 Feb; 130 (2): 241249.

[17] Hackl M, Beyer F, Wegmann K, Leschinger T, Burkhart KJ, Müller LP. The Treatment of Simple Elbow Dislocation in Adults: A Systematic Review and Meta-analysis. Dtsch Arztebl Int. 2015 May; 112 (18): 311-319.

[18] Adas M, Bayraktar MK, Tonbul M, Uzun M, Cakar M, Tekin $\mathrm{AC}$ et al. The role of simple elbow dislocations in cubitus valgus development in children. Int Orthop. 2014 Apr; 38 (4): 797-802. 
[19] Sanchez-Sotelo J, Morrey M. Complex elbow instability: surgical management of elbow fracture dislocations. EFORT Open Rev. 2016 May; 1 (5): 183-190.

[20] Rasool MN. Dislocations of the elbow in children. J Bone Jt Surg (Br). 2004; 86: 1050-1058.

[21] de Haan J, Schep N, Tuinebreijer W, den Harthog D. Complex and Unstable Simple Elbow Dislocations: A Review and Quantitative Analysis of Individual Patient Data. Open Orthop J. 2010; 4: 80-86.
[22] Hay S et al. The Provision of Primary and Revision Elbow Replacement Surgery in the NHS. BESS Surgical Procedure Guidelines. Shoulder and Elbow. 2018; 10 (2): S5-S12.

[23] Doornberg JN, Guitton TG, Ring D. Diagnosis of Elbow Fracture Patterns on Radiographs: Interobserver Reliability and Diagnostic Accuracy. Clin Orthop Relat Res. 2013 Apr; 471 (4): 1373-1378. 